


\title{
Groupes fratries comme dispositifs d'accompagnement des frères et sœurs d'enfants porteurs de handicap et/ou maladie : une revue de la littérature
}

\author{
Support groups for siblings of children with disabilities and/or illnesses: a review of the
}

literature

S. Claudel-Valentin i, D. Arnould ${ }^{\mathrm{ii}}, \mathrm{S}$. Clément-Christophe ${ }^{\mathrm{iii}}$, C. Thomazo ${ }^{\mathrm{iv}}$, C. Dayan ${ }^{\mathrm{v}}$

\section{Résumé}

Cet article propose une revue de littérature sur les groupes de parole à l'attention des frères et sœurs d'enfants porteurs de handicap et/ou maladie. Il s'inscrit dans la continuité des travaux de Dayan, Picon, Scelles et Bouteyre (2006), avec un état des lieux des publications en langue anglophone et francophone parues entre 2006 et 2020. Compte tenu de l'accroissement des études visant les troubles du spectre de l'autisme (TSA), un focus est proposé sur les groupes menés dans ce contexte spécifique. Après avoir décrit la diversité des cadres et approches méthodologiques/ théoriques des groupes fratries, leur mode de fonctionnement et objectifs, une analyse comparative des bénéfices qu'ils apportent est réalisée selon qu'ils concernent tout type de handicap/maladie ou se centrent sur les TSA. Les limites de ces études sont analysées et des perspectives pour des travaux futurs sont énoncés.

\begin{abstract}
This paper is aiming to a literature review on support groups for siblings of children with disability or illness. It is in line with the work of Dayan, Picon, Scelles and Bouteyre (2006), with an inventory of publications in English and French published between 2006 and 2020. Given the increase in studies targeting autism spectrum disorders (ASD), a focus is proposed on groups conducted in this specific context. After describing the diversity of the sibling groups' methodological/theoretical frameworks and approaches, their functioning and objectives, a comparative analysis of the benefits they provide is carried out according to whether they concern any type of disability/disease or focus on ASDs. The limitations of these studies are analyzed and perspectives for future work are outlined.
\end{abstract}

Mots clés : Fratrie ; Handicap ; Maladie ; Groupe de parole ; Enfants.

Keywords: Sibling, Disability ; Illness ; Support group ; Children.

\footnotetext{
' Maître de conférences en Psychologie clinique du développement - Université de Lorraine - 2LPN, EA 7498 - 91 avenue de la Libération, 54000 Nancy - stephanie.claudel-valentin@univ-lorraine.fr

ii Etudiante en Master Psychologie clinique du développement - Université de Lorraine - 23 Boulevard Albert ${ }^{\text {er }}$, 54000 Nancy.

iii Psychologue clinicienne -Institut Médico Educatif Les Orchidées/ Les 3 Tilleuls (AEIM) - 1 rue des Tilleuls, 54720 Chenières.

iv Psychologue clinicienne.

${ }^{\vee}$ Maître de conférences en Psychopathologie - Université Paris Nanterre - CLIPSYD, EA 4430 - 200 avenue de la république, 92000 Nanterre.
} 


\section{Introduction}

Le handicap d'un enfant atteint d'une déficience ou pathologie constitue une épreuve qui rejaillit sur l'ensemble des membres de la famille. La fratrie de l'enfant porteur de handicap/maladie est désormais reconnue par de nombreux chercheurs et praticiens comme une population à risque dont la souffrance psychique peut varier selon l'âge, le rang de naissance, la vulnérabilité des parents et sa propre vulnérabilité (Lefebvre des Noëttes \& Safarty, 2008). L'accompagnement psychologique de la fratrie peut revêtir des formes diverses et contribue à soulager tant les frères et sœurs eux-mêmes que les parents voire l'enfant atteint par son action indirecte sur l'ensemble de la dynamique familiale. Nommés groupes de parole, d'expression, de soutien, ... la mise en œuvre de « groupes fratries » s'accroit depuis une vingtaine d'années. Devant le foisonnement de ces pratiques, Dayan, Picon, Scelles et Bouteyre (2006) ont déjà réalisé un état des lieux des travaux anglophones et francophones publiés sur la question entre 1980 et 2005. Malgré la diversité des dispositifs, aucun axe concret, théorique et méthodologique, n'a été proposé pour s'adapter au contexte de handicap/maladie.

Face à ce constat, nous avons souhaité reconduire cette revue de littérature une quinzaine d'années plus tard pour prendre en compte les évolutions sur la période 2006 - 2020. Pour cela, nous avons consulté plusieurs bases de données électroniques pour accéder à des études scientifiques en utilisant une méthode similaire à celle de Dayan et coll. (2006) à partir des mots clés suivants: "groupe, fratrie, handicap, enfant ; group, siblings, disability, children ». Les bases de données consultées sont: PsychInfo, Eric, Francis, ScienceDirect, Taylor et Francis, Ingentaconnect, Cairn, Pubmed, Springer, Journals.sagepub et Elsevier. Initialement, une trentaine d'articles ont été analysés dont 18 en anglais (d'origine nordaméricaine, australienne, britannique, catalane, grecque, norvégienne, suédoise), et 8 en français (d'origine suisse, belge et française). Parmi ces articles, ont été retenus les travaux ayant fait l'objet d'une évaluation qualitative et/ou quantitative des effets du dispositif étudié, soit un total de 17 articles pris en compte dans cette revue de littérature. Deux revues systématiques portant sur un sujet proche ont par ailleurs été repérées (Hartling, Milne, Tjosvold, Wrightson, Gallivan \& Newton, 2010 ; Tudor \& Lerner, 2015) : ces études soulignent le manque de preuve de l'efficacité des interventions visant le bien-être des fratries et la nécessité de distinguer les conditions de vie des frères et sœurs vivant au contact d'un enfant porteur de handicap mental par rapport à un handicap physique.

$\mathrm{Au}$ cours de ces quinze dernières années, les études s'intéressant spécifiquement aux fratries d'enfants avec troubles du spectre de l'autisme, même si elles restent peu nombreuses, 
ont plus que doublé, passant de 2 études en 2006 à 5 études supplémentaires en 2020. Ce contexte atypique est reconnu comme étant générateur d'un stress majeur chez les parents comparativement à d'autres types de handicap (Hastings 2007 ; Ross \& Cuskelly, 2006 ; Tsao, Davenport \& Schmiege, 2012). La sévérité de l'autisme impacte le vécu des frères et sœurs (Coutelle, Pry \& Sibertin-Blanc, 2011), notamment en termes d'anxiété (Nillama, Derguy, Bellalou \& Cappe, 2019) et peut entraver sa construction identitaire selon les possibilités d'identification-différenciation envers l'enfant porteur de TSA (Claudel, Claudon, Coutelle, 2013 ; Claudel-Valentin, 2018). Compte tenu de la spécificité de ce contexte, les groupes s'adressant aux frères et sœurs d'enfants TSA font l'objet ici d'une attention particulière.

Nous présentons tout d'abord la diversité de ces «groupes fratries » telle qu'elle est décrite dans l'ensemble des publications répertoriées à travers, d'une part, le cadre général qu'elles proposent et d'autre part, la démarche expérimentale ou thérapeutique privilégiée. Une synthèse du fonctionnement et des objectifs définis dans chacune de ces recherches est réalisée en prenant en compte la population, la temporalité, le lieu, les médias utilisés et les thèmes abordés. Nous procédons ensuite à une analyse comparative des effets de ces groupes selon qu'ils concernent tout type de handicap/maladie ou qu'ils sont centrés sur le contexte particulier des TSA. Pour finir, nous proposons une discussion ouvrant sur de nouvelles perspectives de recherche.

\section{Diversité des groupes}

Chaque publication retenue a fait l'objet d'une analyse détaillée permettant notamment de distinguer les modèles associés aux interventions et leur protocole d'évaluation (cf. tableau récapitulatif en annexe).

\subsection{Indications, cadre et objectifs des groupes}

Le cadre des groupes fratries étudiés revêt des configurations différentes et répond à des objectifs variés. Les dispositifs ont toutefois en commun la volonté d'offrir un espace d'expression et d'apporter des éléments de connaissance ou de compréhension sur la situation de handicap/maladie du frère ou de la sœur.

Le cadre de ces dispositifs est construit généralement à partir d'une pratique clinique et/ou de travaux de la littérature scientifique, et s'inscrit le plus souvent dans une perspective de prévention. Il s'appuie sur le constat de difficultés d'adaptation de la fratrie à la situation de handicap/maladie pouvant générer différents symptômes tels que l'hypermaturité, la 
dépression, l'inhibition intellectuelle, l'échec scolaire ou le sentiment d'abandon. Il prend en compte la variabilité des affects de la fratrie qui peut être au prise avec des sentiments de tristesse, honte, culpabilité sans parvenir à en parler, notamment en famille (Scelles, Bouteyre, Dayan \& Picon, 2012). Ces dispositifs sont proposés lorsque le handicap du frère ou de la sœur est avéré et diagnostiqué ou quand le handicap devient la conséquence d'une maladie dépistée dont on ne peut anticiper l'évolution. La participation précoce à un groupe fratrie vise à limiter les répercussions psychologiques de la situation de handicap/maladie sur la fratrie dans le sens où l'inscription du sujet dans le groupe peut lui permettre de faire face au traumatisme vécu et à ses éventuelles conséquences (Milman, 2007). De manière plus interventionniste, le cadre dans certaines études s'organise parfois selon des objectifs plus précis dans le but de promouvoir le fonctionnement émotionnel et comportemental des frères et sœurs (Roberts, Ejova, Giallo, Strohm, Lillie \& Fuss, 2015). Ces objectifs visent notamment l'augmentation des connaissances des frères et sœurs au sujet du handicap, le développement d'un réseau de pairs et/ou l'amélioration des interactions entre frères et sœurs (Granat, Nordgren, Rein \& Sonnander, 2012 ; McCullough \& Simon, 2011).

Les publications les plus récentes révèlent une ouverture du cadre à partir de supports variés. Le recours à l'environnement numérique a par exemple été exploré pour faciliter l'accès à un réseau de frères et sœurs malgré l'isolement géographique de certains (Tichon, 2015). Dans le même sens, un système d'audioconférence avec quelques regroupements en présentiel a également été proposé pour engager des groupes de soutien entre frères et sœurs (Gettings, Franco \& Santosh, 2015). Des thérapies artistiques ont par ailleurs été mises en place dans le but de favoriser l'expression de soi au-delà de la parole et ainsi appréhender la réalité différemment (Wallace, Packman, Huffman, Horn, Cowan, Amylon \& Moses, 2014). Des camps d'été ont également vu le jour dans le but d'accompagner les fratries entières à un niveau psychosocial et offrir de surcroît un temps de répit aux parents $(\mathrm{Wu}$, Prout, Parikshak \& Amylon, 2011). Même si ces dispositifs novateurs s'éloignent sensiblement des «groupes fratries » tels qu'ils ont pu être conçus initialement, ils ont pour point commun de permettre aux frères et sœurs de prendre conscience que d'autres enfants vivent des situations analogues, de mutualiser leurs expériences et de s'adapter au quotidien.

\subsection{Des approches épistémologiques variées}

Initialement, les études des groupes fratries ont été élaborées dans une démarche expérimentale à visée psychoéducative. Il s'agit principalement de dispositifs anglo-saxons 
créés à des fins de recherche. Les travaux français, apparus plus tardivement, évoquent quant à eux des dispositifs mis en place par des équipes de soins dans une démarche clinique à visée thérapeutique (Dayan et al., 2006). Des méthodologies mixtes tendent toutefois à se développer avec une prise en compte de données qualitatives dans certaines recherches expérimentales et une tentative d'objectivation des données dans certaines recherches cliniques pour rendre compte des effets observés.

\subsubsection{Groupes mis en place dans le cadre d'une démarche expérimentale}

La littérature anglo-saxonne publiée depuis 2006 fait référence quasi systématiquement à des groupes fratries s'inspirant de théories cognitivo-comportementales et s'inscrivant dans une démarche de recherche empirique.

Ces études s'attachent à un aspect particulier du vécu des fratries et visent le changement de comportements et d'affects des enfants à l'égard de leur frère ou sœur en situation de handicap/maladie par une intervention d'ordre psychoéducative sur une période prédéterminée généralement courte (Brouzos, Vassilopoulos \& Tassi, 2017 ; Granat, Nordgren, Rein \& Sonnander, 2012). Elles soulignent l'intérêt de fournir des informations sur le handicap aux frères et sœurs afin de leur permettre de développer des stratégies adéquates pour mieux vivre avec leur pair porteur de handicap/maladie (Granat, et al., 2012 ; McCullough \& Simon, 2011). Ces connaissances apprises visent à apporter des éléments de compréhension et de résolution des problèmes rencontrés en famille. Le groupe est vu comme un lieu d'apprentissage et il s'agit de mettre en place un programme de soutien et d'acquisition de compétences (Cooke \& Semmens, 2010 ; Kryzak, Cengher, Feeley, Fienup \& Jones, 2015). Ces études mettent par ailleurs l'accent sur l'intérêt de la communication émotionnelle au sein des groupes fratries (Vatne \& Zahl, 2017).

Les travaux ayant recours au support numérique s'inscrivent dans cette approche expérimentale. Il s'agit d'identifier le rôle spécifique de la technologie dans la communication et le partage des réflexions et des sentiments sensibles propres aux fratries en situation de handicap/maladie (Gettings et al., 2015 ; Tichon, 2015). De même les dispositifs de camps de vacances pour les enfants en situation de maladie et leurs fratries sont construits selon un modèle psychoéducatif dont les effets bénéfiques au niveau de la famille entière ont pu être vérifiés (Wu et al., 2011).

Dans ces études, l'évaluation des effets de la participation au groupe est systématisée et armée avec recours à des questionnaires, échelles de mesure voire entretiens semi-directifs, 
proposés le plus souvent juste avant et juste après l'intervention (mesures auto et hétérodéclaratives auprès des parents). Un système d'analyse de contenu des échanges au sein du groupe avec prise en compte de données tant qualitatives que quantitatives est parfois établi pour témoigner des sujets abordés et du type de soutien social mis en place (Tichon, 2015 ; Vatne, 2007). Des observations comportementales des interactions entre frères et sœurs avant et après le groupe sont parfois réalisées en codant notamment des enregistrements vidéos de dyades frères et sœurs jouant ensemble pour rendre compte de l'évolution de l'ajustement mutuel (Kryzak et al., 2015). Les sondages de satisfaction peuvent comporter des questions ouvertes pour intégrer les expériences sociales vécues (Kryzak et al., 2015 ; Wu et al., 2011). Ainsi, l'apport de données qualitatives tend à être reconnu comme un soutien complémentaire dans le travail d'analyse (Tichon, 2015).

\subsubsection{Groupes mis en place dans le cadre d'une démarche clinique et thérapeutique}

De plus en plus de structures de soins dans le secteur médico-social (CAMSP, SESSAD, etc.) ou hospitalier (centre de soins, hôpital d'enfants, CMP, etc.) proposent des groupes fratries dans une démarche clinique et/ou thérapeutique. Les travaux francophones rendent compte de ces expériences menées le plus souvent dans une approche psychodynamique et/ou systémique.

L'accent est porté ici sur l'expression du sujet, la mise en mots de son vécu singulier. Le nombre de séances n'est pas prédéterminé et le contenu est défini par les participants euxmêmes. Le groupe a une fonction d'étayage et de contenance et peut avoir des effets thérapeutiques (Scelles et al., 2012). L'approche groupale se fonde sur la référence à l'inconscient, individuel et groupal (Milman, 2007). L'espace de parole offert aux frères et sœurs est considéré comme une opportunité de s'identifier à d'autres vivant un quotidien similaire et éprouvant des sentiments communs, généralement enfouis et inhibés (Valls, 2009) et vise l'élaboration des angoisses, sentiments de jalousie, culpabilité (Despars, Jaunin, Monnier, Wannaz, Vernez, Tolsa, Ansermet, \& Müller-Nix, 2013). Les jeux d'alliance entre enfants et l'effet mirroring sont le moteur de la dynamique groupale (Dayan \& Scelles, 2017). Cet effet de miroir « rassemble l'empathie envers l'autre » dans le respect de sa différence et de son altérité. Il permet une uniformité entre les membres du groupe et donne « accès au sens inconscient de ce qui est communiqué »(Knauss, 2009, p.35).

Fondée sur une épistémologie de l'intersubjectivité, l'appréciation des effets de la participation au groupe fratrie dans cette approche se fait généralement dans l'après coup par une clinique le plus souvent non armée, sur la base des observations menées au fil des séances de groupe fratrie. Le sujet est pris en compte dans sa singularité et dans sa relation avec son 
environnement. Le mal-être identifié peut venir de perturbations psychiques ou adaptatives, indépendantes de la situation de handicap/maladie. L'évaluation prend en compte les évolutions en termes de maturité, sensibilité, attention aux autres, capacité de tolérance, qui peuvent être directement observables à travers la dynamique de groupe. Toutefois, les études tendent à intégrer également de plus en plus le point de vue des parents par un entretien clinique préalable et un entretien de suivi dans le but à la fois d'assurer une alliance thérapeutique parentale et d'objectiver l'évaluation des effets observés (Dayan et al., 2017 ; Valls, 2009).

\section{La construction des études}

\subsection{Population}

\subsubsection{Pathologies concernées}

Les études portant sur les groupes fratries publiées depuis 2006 concernent une grande variété de situations de frères et sœurs d'enfants « à besoins spéciaux » selon les termes de Tichon (2015). En France dans la majorité des cas, les groupes fratries sont l'initiative d'un centre de soins et intègrent des frères et sœurs d'enfants ayant différents types de handicap/maladie (Scelles et al., 2012 ; Dayan et al., 2017). Dans les études anglo-saxonnes, le recrutement des familles peut s'effectuer par diffusion d'annonces ou courriers par les investigateurs d'études et de dispositifs (Wu et al., 2011 ; Gettings et al., 2015 ; Roberts et al., 2015 ; Tichon, 2015 ; Vatne \& Zahl, 2017). Une étude regroupe les frères et sœurs par type de pathologies de l'enfant atteint après avoir procédé au recrutement de l'ensemble des sujets (Granat et al., 2012). D'autres études proposent un dispositif à des frères et sœurs d'un enfant à pathologie spécifique comme le syndrome de Down (Valls, 2009), les troubles du spectre de 1'autisme (Brouzos et al., 2017 ; Cooke \& Semmens, 2010 ; Jones et al., 2020 ; Kryzac et al., 2015 ; McCullough \& Simon 2011). Certaines se sont élargies à l'accueil particulier aux familles et fratries d'enfants prématurés (Despars et al., 2013), d'enfants atteints de cancer (Milman, 2007 ; Wallace et al., 2014 ; Wu et al., 2010).

Les recherches intègrent parfois un groupe témoin sans intervention spécifique (Wallace et al., 2014 ; Roberts et al., 2015 ; Brouzos et al., 2017), ou avec intervention alternative pour contrôler tout facteur thérapeutique non spécifique (Jones et al., 2020).

\subsubsection{Taille des groupes et caractéristiques morphologiques}


La taille des échantillons ne figure pas toujours dans les articles. Dans une approche clinique, de petits groupes sont privilégiés, les dispositifs sont le plus souvent conçus pour accueillir de 3 à 10 enfants. Les groupes s'inscrivant dans une approche expérimentale sont généralement plus conséquents et peuvent compter une cinquantaine de participants.

La constitution des groupes est essentiellement établie en fonction de l'âge des participants. Celui-ci peut s'échelonner de 3 à 18 ans. Des sous-groupes sont parfois proposés pour tenir compte des besoins différenciés selon l'âge des participants (Brouzos et al, 2017 ; Milman, 2007 ; Valls, 2009). Dans les dispositifs à destination d'enfants, le groupe revêt essentiellement une fonction d'étayage, alors que dans les dispositifs s'adressant à des adolescents il peut avoir davantage une fonction d'aide à la construction identitaire.

Certains dispositifs intègrent plusieurs membres d'une même fratrie (Cooke et al., 2010 ; Roberts et al., 2015), voire vont jusqu'à inclure l'enfant en situation de handicap (Jones et al., 2020 ; Kryzak et al., 2015 ; Wu et al. 2011).

\subsection{Structure et fonctionnement des groupes}

\subsubsection{Temporalité}

Les groupes présentés se réunissent le plus souvent selon une périodicité régulière, de façon hebdomadaire, mensuelle, bimensuelle, un mois sur deux, mais aussi sous forme de session sur quelques mois (7-8 semaines), une semaine entière, ou encore sous forme de rencontres ponctuelles à intervalles prédéfinis. Dans le cas où les séances de groupe s'étendent sur une longue période, il importe que la régularité et la stabilité temporelles soient respectées pour assurer un cadre contenant. Les études anglo-saxonnes privilégient les sessions. La durée, qui n'est pas toujours précisée, oscille selon les dispositifs entre une et deux heures avec une pause. Le moment dépend des dispositifs : mercredi, samedi, en fin de journée (après école) ou encore durant les vacances d'été.

\subsubsection{Lieu}

En France, la plupart des rencontres en groupe se déroule sur le lieu d'accompagnement et de soins du frère porteur de handicap. Dayan et al. (2017) se sont interrogés au sujet de la pertinence de confondre lieu de soin de l'enfant porteur de handicap (TSA) et lieu de regroupement des frères et sœurs (période de latence). L'expérience a au contraire enthousiasmé les frères et sœurs qui ont manifesté une motivation mêlée d'identification de 
rivalité, et un intérêt pour ce lieu réservé au frère TSA. De même, les centres hospitaliers, les camps d'été entrent dans le principe de partage du même lieu.

Dans une démarche de recherche, le groupe peut être proposé sur des sites universitaires (Jones et al., 2020 ; Kryzac et al., 2015). Dans les études non francophones, ces groupes ont parfois lieu dans les locaux d'associations ou d'ateliers particuliers (Valls, 2009 ; McCullough \& Simon, 2011 ; Cooke \& Semmens, 2010 ; Brouzos, et al., 2017). Il arrive que le choix soit laissé aux familles pour que l'espace soit le plus adapté possible (Wallace et al., 2014). Cooke \& Semmens (2010) mettent en avant le bénéfice de lieux offrant un grand espace pour l'animation des groupes, l'atout de la présence d'un jardin pour les pauses et la possibilité d'avoir une salle séparée permettant aux parents de rencontrer d'autres parents, sur place, dans un climat accueillant et détendant.

\subsubsection{Mise en place du dispositif}

La procédure de mise en place de groupes fratries est décrite à travers les différents articles selon les étapes suivantes : une phase de recrutement avec ou sans entretien préalable à l'entrée dans le groupe, la mise en œuvre des séances, une phase d'évaluation des effets de la participation aux groupes. Lorsqu'un entretien préalable est proposé, celui-ci s'adresse aux parents voire à l'enfant concerné. Il s'agit de présenter le cadre ou le programme, d'expliquer les objectifs et les modalités de fonctionnement du dispositif. Dans une visée de recherche, un formulaire de consentement est demandé précisant les conditions du dispositif(volontariat, liberté, respect, confidentialité, respect de soi et des autres). Cet entretien, plus ou moins structuré, permet d'évaluer la pertinence de la participation au dispositif et d'analyser la situation initiale de la famille / fratrie. Des outils standardisés peuvent être utilisés aux différentes étapes du dispositif pour évaluer des caractéristiques ciblées telles que la qualité des relations fraternelles, les troubles comportementaux et émotionnels, l'adaptation, l'anxiété, l'estime de soi, le soutien social perçu (cf. tableau en annexe).

Le contenu des séances en elles-mêmes est rarement détaillé. Seuls 5 articles apportent des renseignements sur les séquences d'une séance (Brouzos, 2017 ; Dayan et al., 2017 ; Despars et al., 2013 ; Krysac et al., 2015 ; Valls, 2009). Après le premier contact, ces séances sont alors découpées le plus souvent en 4 temps :

1) Présentation initiale des différents membres du groupe, des animateurs, des modalités du groupe (programme et règles de fonctionnement);

2) Séquence 1 : amorçage des échanges avec information du sujet ou thème du jour ; 
3) Séquence 2 : mise en activité à partir de médias variés ou sur la base de programmes existants (activités plus ou moins structurées pour s'ajuster aux besoins des participants) ;

4) Séquence $3:$ Discussion avec partage réciproque des expériences;

5) Séquence 4 : clôture de la séance.

Les thématiques abordées sont soit orientées par le discours des participants (Dayan et al., 2017), soit prédéfinies, comme dans les approches TCC (Roberts et al., 2015) ou dans les programmes psycho-éducatifs (Cooke \& Semmens, 2010).

Les thématiques les plus souvent abordées peuvent être regroupées en six domaines :

1) la dynamique familiale à travers son fonctionnement, le vécu quotidien, le degré de communication ;

2) la souffrance des parents, le sentiment d'impuissance et le sentiment d'être oublié ;

3) la relation de la fratrie : l'ambivalence, la rivalité, l'agressivité inhibée ou déplacée ;

4) les informations sur la pathologie et la manière d'en parler autour de soi ;

5) la question du regard des autres et celle du soutien social ;

6) la perception de soi (individualisation, vie affective et émotionnelle, gestion du stress et adaptation, projection dans l'avenir).

Le partage d'un goûter est parfois mentionné (en cours ou fin de séance). Les auteurs ne proposent pas d'analyse de sa fonction ; on connaît pourtant l'importance des rituels dans les groupes thérapeutiques et de soutien.

La clôture de fin de séance est comptée comme un temps à part entière : le groupe apprend comment bien se séparer après avoir revisité le sujet évoqué et posé des questions si nécessaire. C'est aussi le moment réservé à l'appréciation de la séance et l'annonce de la suivante. Le départ est concrétisé par les salutations entre participants.

En continuité des séances, des actions concrètes en famille sont parfois proposées. Elles permettent une intégration approfondie des moyens élaborés durant l'atelier et l'information des parents au sujet de facteurs favorisant l'évolution et le bien-être de leur enfant. Dayan et al. (2017) proposent un retour aux parents sur les intérêts ou les limites de la participation au groupe. En fonction des besoins repérés, des suivis individuels peuvent être préconisés à l'issue des séances (Valls, 2009).

La majorité des groupes de soutien ouverts aux fratries est co-animée par des professionnels formés et qualifiés : des psychologues, des psychothérapeutes, des éducateurs spécialisés, des psychomotriciens, des art-thérapeutes ou des personnes de services hospitaliers. Milman (2007) précise l'intérêt de la co-animation par un couple homme-femme pour refléter 
le mode de fonctionnement familial. La fonction des animateurs est de garantir le cadre, c'està-dire la tenue et la durée de la séance, et de favoriser la circulation de la parole. Dans ce rôle de médiateur qui explore les représentations et les émotions et stimule les échanges, l'animateur se garde de tout conseil ou opinion. Une seule étude précise que l'animateur psychologue peut être assisté d'un étudiant en psychologie. Elle mentionne la présence d'un psychologue superviseur et celle d'un chercheur observateur durant les séances et des temps de débriefing entre animateurs (Roberts et al., 2015).

\section{Résultats des recherches}

Quels que soient le type de handicap/maladie pris en compte, le type de démarche mis en œuvre, les modalités d'évaluation du dispositif, les recherches révèlent des résultats positifs à la participation à un groupe fratrie, même s'ils sont parfois nuancés. Compte tenu de la diversité des modèles des études, les résultats recensés sont présentés ici de manière thématique. Ces résultats rejoignent les conclusions des recherches antérieures et apportent de nouvelles données grâce à des dispositifs innovants (dispositifs à distance, incluant l'ensemble des membres de la famille, etc.).

Dans le contexte TSA, des spécificités inhérentes aux caractéristiques de la pathologie sont observées dans le fonctionnement des groupes fratries, ce qui vient appuyer l'intérêt de créer des dispositifs adaptés au contexte de handicap considéré.

\subsection{Des effets positifs quelle que soit la situation de handicap/maladie}

Que le groupe se constitue par rapport à la spécificité du handicap / de la maladie ou qu'il accueille des frères et sœurs confrontés à des situations de natures différentes, les effets de la participation au groupe fratrie s'avèrent bénéfiques.

\subsubsection{Un espace de parole qui éclaire}

En premier lieu, les auteurs soulignent que le groupe fratrie offre un espace extérieur à la cellule familiale qui donne une place spécifique au frère ou à la sœur de l'enfant porteur de handicap/maladie (Despars et al., 2013). Le frère ou la sœur peut se sentir reconnu(e) pour ce qu'il/elle est, c'est à dire un être à part entière et non seulement comme « frère ou sœur de ... » (Scelles et al., 2012). Le groupe permet de « conscientiser» le handicap et de s'exprimer plus clairement sur son expérience. Plus la pathologie est comprise mieux elle est intégrée, et l'expression des émotions et sentiments s'avère plus aisée. Cet espace éclaire le participant sur 
l'impact du handicap dans son existence, tout comme le contenu des échanges éclaire la recherche et la pratique et permet de mieux comprendre les besoins de la fratrie (Dayan et al., 2017).

\subsubsection{Des émotions et sentiments mis au grand jour}

Au-delà de l'ambivalence naturelle dans les relations humaines et en particulier fraternelles, en contexte de handicap/maladie, les émotions négatives bien que fréquentes sont exprimées avec plus de retenue et nuance. L'influence de l'éducation parentale semble jouer dans la tolérance envers le frère différent (Tichon, 2015). Comme le soulignent de nombreuses études, la jalousie est générée par la frustration liée à la moindre disponibilité parentale et aux différences de traitements entre le frère et le reste de la fratrie (Dayan et al., 2017 ; Despars et al., 2013 ; Scelles et al., 2012). La colère est rarement explicite mais l'impuissance et l'incapacité de faire face aux comportements parfois même agressifs du frère sont clairement signifiées. Les frères et sœurs rapportent peu fréquemment mais en détails ces agressions physiques et expriment combien faire des concessions a un impact fort sur leur équilibre émotionnel (Tichon, 2015 ; Vatne \& Zahl, 2017 ; Valls, 2009). La détresse des frères et sœurs face à ces comportements est telle qu'ils peuvent fantasmer un retour à la normalité ou souhaiter un autre handicap du frère «moins violent » (Tichon, 2015). Despars et al. (2013) soulignent une différence dans l'expression des émotions selon l'âge : les sentiments dits «mitigés » sont observés chez tous, tandis que « des mouvements identificatoires à l'égard des parents sont plus fréquents et plus marqués » chez les 7-8 ans (p. 240). La fonction d'étayage du groupe rend possible l'aveu des émotions majoritairement négatives et permet aux frères et sœurs de partager leurs sentiments (Valls, 2009), de recevoir le soutien des adultes encadrants et celui des pairs vivant une situation similaire (Wu et al., 2011).

\subsubsection{Des effets aux niveaux personnel, familial, social}

Les effets thérapeutiques observés dépendent de la solidité du cadre et des processus initiés par la dynamique du groupe. Ces effets peuvent s'étendre à la sphère familiale et à la vie sociale.

Des effets thérapeutiques significatifs sur la culpabilité sont observés chez les frères et sœurs participants. Cette culpabilité tend à s'atténuer et à être dédramatisée lors de la découverte d'éprouvés similaires chez les pairs ; on reconnaît ici l'effet miroir repéré par les 
thérapeutes de groupes d'enfants (Despars et al., 2013). L'expérience groupale remplace ainsi un sentiment de solitude par un sentiment d'appartenance (Milman, 2007 ; Wu et al., 2011).

Le travail d'élaboration permis par le groupe favorise une amélioration du fonctionnement émotionnel et comportemental des frères et sœurs. Une progression dans leur capacité d'adaptation, dans la résolution de problèmes et dans leur perception du soutien social a été constatée en croisant le regard des parents, des enseignants et des pairs (Roberts et al., 2015). Ainsi, les frères et sœurs développent un sentiment de contrôle et parviennent à une meilleure gestion de leurs émotions au quotidien. Dans un contexte de cancer infantile, l'intervention a pu notamment induire chez les frères et sœurs une prise de conscience de leur rôle dans le traitement de leur pair et par là-même diminuer leur sentiment d'impuissance (Wallace et al., 2014).

La participation au groupe contribue à développer également des compétences sociales et créatives (Wu et al., 2011). Les nouveaux dispositifs offrent des opportunités d'ouverture sociale, en particulier dans les groupes en ligne, les camps d'été et l'art-thérapie (Gettings et al., 2015 ; Roberts et al., 2015 ; Tichon, 2015 ; Wu et al., 2011 ; Wallace et al., 2014).

Ces effets thérapeutiques ne se cantonnent pas au mieux-être du frère ou de la sœur mais s'étendent aux relations familiales. L'évolution de la représentation du handicap permet aux frères et sœurs de changer leurs comportements, d'être plus affirmés, moins agressifs voire plus proches du pair handicapé (Valls, 2009). Dans un second temps, la communication en famille apparaît nettement améliorée (Gettings et al., 2015) et les frères et sœurs osent s'enquérir d'informations sur la pathologie ou leur histoire (Dayan et al., 2017). La culpabilité tend à s'apaiser également du côté des parents. La prise en compte du vécu parental dans le dispositif (entretien familial, groupe parents) favorise la dynamique familiale et fait partie des facteurs à considérer pour assurer le maintien des effets du groupe fratrie (Scelles et al., 2012).

\subsubsection{Validité sociale et limites des groupes}

Lorsque les frères et sœurs sont interrogés sur leur appréciation du dispositif, ils se montrent généralement très satisfaits de ce qui leur a été proposé (Roberts et al., 2015 ; Wu et al., 2011). Wu et al., (2011) rapportent que la variété des activités selon la nature du dispositif, l'atmosphère de soutien ressenti, l'opportunité de s'extraire du quotidien sont les points positifs soulignés tant par les participants que par les parents. Les thérapies artistiques s'avèrent pertinentes pour communiquer sans être contraint de parler (Wallace et al., 2014). Les modes innovants de groupe de soutien en ligne ont permis de dépasser les limites géographiques et de 
donner au plus grand nombre l'accès à la plateforme d'expression et d'échanges (Gettings et al., 2015). Il est intéressant de souligner que les propositions de dispositifs rassemblés sur un temps concentré sont bien appréciées.

Les résultats de ces expériences de groupes fratries font émerger toutefois des limites à considérer dans les futures initiatives d'aide aux fratries en contexte de handicap. Certains résultats peuvent apparaître contrastés d'une étude à l'autre comme par exemple l'influence sur le degré d'estime de soi et l'anxiété, variable selon le cadre de l'intervention et le protocole d'évaluation (Kryzac et al., 2015 ; Wallace et al., 2014). La durée des interventions par ailleurs viendrait expliquer que les effets observés ne sont parfois pas maintenus dans le temps (Valls, 2009). A noter que lorsque les études prennent en compte la pérennité des effets, l'évaluation ne dépasse cependant pas 3 à 6 mois après l'intervention (Gettings, 2015 ; Roberts et al., 2015). Les bénéfices du groupe dépendent aussi de la spécificité de la dynamique familiale, des modèles de communication familiaux, du degré de sévérité du handicap et du niveau de stress parental (McCullough \& Simon, 2011). Aussi, la mise en œuvre d'études longitudinales serait nécessaire pour rendre compte des trajectoires développementales et des possibilités de résilience des frères et sœurs.

\subsection{Spécificités dans le contexte des TSA}

Au vu de l'accroissement des études portant sur les groupes fratries dans le contexte des TSA, nous avons souhaité effectuer un focus sur la nature des effets dans ce contexte spécifique. Les troubles du spectre de l'autisme (TSA) ou autisme (traduction française du DSM), selon sa nomination dans les différents articles, sont toujours définis par des traits communs relatifs à la nomenclature du DSM V d'une part, et par des observations cliniques communes quelles que soient les sources des recherches d'autre part. Ainsi, l'autisme affecte les comportements humains essentiels tels que la capacité de communiquer, d'interagir avec ses pairs et l'environnement de manière générale. Les capacités d'adaptation sont affectées par un mode de compréhension atypique, des particularités sensorielles et des déficits plus ou moins marqués concernant les compétences cognitives. Le langage est souvent affecté et la présence de comportements restreints et répétitifs associés à un besoin d'immuabilité important impacte de manière certaine la dynamique familiale.

\subsubsection{Diversité des modèles d'intervention}


Nous avons relevé cinq études en langues anglophones publiées récemment, au Royaume-Uni (Cooke \& Semmens, 2010), en Grèce (Brouzos et al, 2017) et aux Etats-Unis (Jones et al., 2020 ; Kryzac et al., 2015 ; McCullough \& Simon, 2011).

Il s'agit principalement de groupes de soutien menés dans une approche psychoéducative, qui s'intègrent selon le cas dans des modèles d'intervention incluant l'enfant porteur de TSA (Jones et al., 2020 ; Kryzac et al., 2015). Les objectifs généraux sont d'améliorer l'adaptation sociale et émotionnelle des frères et sœurs par le biais de discussions formelles et informelles, en passant parfois par des lectures axées sur l'information et l'apport de connaissances sur les TSA (Kryzak et al., 2015). Ces groupes permettent d'aborder les problèmes spécifiques auxquels les enfants atteints d'autisme sont confrontés et d'élaborer des stratégies efficaces pour composer avec eux (Cooke \& Semmens, 2010). Des programmes de formation très structurés pour faire face à cette situation fraternelle singulière peuvent être mis en place en tenant compte de l'âge de développement des frères et sœurs (Brouzos et al., 2017). La nécessité de prendre en compte le degré de sévérité des TSA peut également être soulignée pour la constitution du groupe fratrie (Kryzak, 2015). Dans les dispositifs intégrant l'enfant TSA, deux étapes sont généralement identifiées avec d'abord des espaces différenciés, sous la forme d'un groupe de compétences sociales à destination de l'enfant porteur de handicap et d'un groupe de soutien pour les frères et sœurs, puis avec un espace commun récréatif visant la relation fraternelle et la communication familiale. Un groupe contrôle, centré uniquement sur les capacités d'attention des frères et sœurs, peut être proposé pour rendre compte des effets thérapeutiques du groupe de soutien (Jones et al., 2020).

\subsubsection{Les effets spécifiques repérés}

L'accent est ici porté de manière saillante sur l'intérêt de développer des connaissances spécifiques sur les TSA pour améliorer la compréhension du vécu de l'enfant atteint, favoriser les interactions fraternelles et l'ouverture sociale (Kryzak et al., 2015 ; McCullough \& Simon, 2011). L'étude de Brouzos et al. (2017) démontre une réduction significative des difficultés d'adaptation et problèmes émotionnels et comportementaux des frères et sœurs à l'issue de la participation au groupe de soutien comparativement à un groupe témoin. Il en est de même en comparaison avec un groupe contrôle centré sur l'attention (Jones et al., 2020). Les symptômes d'anxiété et de dépression chez les frères et sœurs tendent à diminuer, de manière d'autant plus marquée que les troubles de l'enfant autiste sont sévères (Jones et al., 2010). Le partage de solutions entre frères et sœurs permet d'élaborer des stratégies communes pour faire face au 
comportement de l'enfant TSA (McCullough \& Simon, 2011). Dans un contexte de groupe intégrant plusieurs types de handicap/pathologie (Granat et al., 2012), les frères et sœurs d'enfants TSA tireraient en revanche moins de bénéfices à la participation au groupe que les autres membres en raison des défis uniques auxquels ils sont confrontés.

Cette particularité "des comportements-défis" spécifiques aux TSA est une dimension remarquable qui fait l'objet de discussions prégnantes dans le cadre des interventions centrées sur l'autisme. La dynamique de groupe tend à favoriser la mise en lien entre comportements problème, manque de compétences en communication, difficultés d'intégration sensorielle ou de transition ; ce qui peut permettre, comme en témoignent McCullough et Simon (2012) dans leur recherche, aux membres du groupe «de dépersonnaliser l'agressivité de leurs frères et sœurs et de l'éviter ou de la contenir quand elle se produit » (p.325). Aussi l'attitude des parents face à ces comportements défis, la manière dont ils les comprennent et les gèrent, influence la relation fraternelle. Lorsqu'ils parviennent à contenir la violence de l'enfant porteur de TSA, le lien est ainsi mieux préservé.

\section{Préconisations et perspectives de recherche}

Cette revue de littérature internationale nous permet d'avoir un large panorama des pratiques récentes de «groupes fratries ». La tendance relevée en 2006 par Dayan et ses collaborateurs est toujours actuelle avec une disparité des approches entre les travaux anglophones et francophones. Les résultats dans l'ensemble des études sont positifs et globalement similaires aux précédents travaux malgré l'hétérogénéité des modèles utilisés. Toutefois certaines limites dans la construction des études sont à souligner et nous amènent vers d'autres préconisations et pistes de recherche.

\subsection{Limites et préconisations}

Les principales limites ont trait au recrutement, à la constitution des groupes, à la taille des échantillons, aux outils d'évaluation utilisés, à la durée des dispositifs et aux données de suivi, mais aussi au manque d'ancrage théorique de certaines interventions.

\subsubsection{Recrutement}

Dans de nombreuses études, le recrutement a constitué un défi au vu de la non reconnaissance chez les parents de l'intérêt de prendre en compte le vécu des frères et sœurs. Des résistances ont pu être observées face au risque de faire émerger certaines émotions 
pouvant mettre à mal le fragile équilibre familial (McCullough \& Simon, 2012). La moindre disponibilité des parents, notamment lorsque les enfants sont en bas âge et que le handicap est important, a également été invoquée au vu des contraintes organisationnelles de la famille.

Aussi, les critères d'inclusion/exclusion dans le recrutement sont rarement mentionnés ou demeurent imprécis. Ceux qui figurent le plus souvent sont les signes de souffrance psychique et le besoin de s'exprimer sur la situation de handicap. Ces critères nécessiteraient d'être davantage considérés pour définir les conditions adaptées à la participation au groupe selon les besoins des frères et sœurs.

Par ailleurs, des biais liés au volontariat des participants (familles engagées, enfants motivés) doivent être pris en compte dans l'analyse des effets des dispositifs (Granat et al., 2012).

\subsubsection{Constitution des groupes}

L'absence de prise en compte du critère de l'âge dans la constitution du groupe peut interférer sur son fonctionnement. Dans l'étude de Brouzos et al. (2017), les comparaisons entre les groupes d'âge ont révélé que les enfants étaient plus susceptibles de bénéficier de l'information fournie sur le handicap, tandis que les adolescents étaient plus touchés par les activités de groupe qui ciblent leurs difficultés d'adaptation. Ainsi il apparaît essentiel de constituer les groupes en tenant compte des besoins de développement spécifiques aux groupes d'âge.

La nature du handicap considéré mais aussi le degré de sévérité des symptômes de l'enfant porteur de handicap nécessiteraient d'être pris en compte. Brouzos et al. (2017) dans un contexte de TSA préconisent d'inclure des situations d'autisme variables (de légers à plus graves) afin de faciliter de « riches discussions de groupe et d'obtenir un meilleur soutien parmi les membres du groupe » (p. 279). De même, Tudor \& Lener (2015), à l'issue de leur revue de littérature, préconisent de prendre en compte le niveau de comportement problématique, la gravité des symptômes du frère/de la sœur affecté(e), ainsi que le niveau de détresse psychologique des parents pour pouvoir mesurer plus spécifiquement les effets des groupes en fonction de la situation individualisée de chacun des participants.

A noter que la régularité des participants peut présenter un obstacle à la constitution du groupe dans la durée. Le choix d'intégrer le groupe n'est pas toujours personnel mais correspond davantage à une demande des parents. Ainsi il peut y avoir « une phase de pré- 
affiliation à établir qui sera décisive dans la régularité des participations futures » (BermanRossi, 1993, cité par McCullough \& Simon, 2011, p. 323).

\subsubsection{Taille du groupe}

La taille du groupe, trop petit ou trop grand, est souvent évoquée dans les limites, elle modifie la dynamique de groupe et reste une variable des effets du groupe. Certains auteurs notamment dans le contexte des TSA, recommandent de maintenir la taille du groupe en dessous de 8 membres pour faciliter le traitement des activités et l'émergence des facteurs thérapeutiques. Selon eux « vivre avec un frère ou une sœur atteint de TSA place les jeunes à un niveau de stress personnel inhabituel de sorte qu'ils ont besoin de plus d'espace pour se sentir à l'aise et s'exprimer »(Brouzos et al., 2017, p.280).

Le fonctionnement en groupe ouvert ou groupe fermé est par ailleurs rarement mentionné par les auteurs. Seules 2 études spécifient avoir recours à un groupe ouvert (Milman, 2007 ; Dayan et al., 2017) sans toutefois justifier leur choix et discuter l'intérêt ou les limites de cette modalité sur le développement des processus thérapeutiques.

\subsubsection{Outils d'évaluation}

Les limites liées aux biais de l'évaluation, à la fidélité des outils utilisés sont les plus souvent rapportées. La nécessité de croiser les sources d'information et la nature des données est de rigueur. A titre d'exemple, Roberts et al. (2015) ont pu noter des discordances entre autoévaluation des frères et sœurs, hétéro-évaluation des parents et observation directe concernant le fonctionnement émotionnel et comportemental de la fratrie au fil du dispositif. Les auteurs ont émis l'hypothèse que les frères et sœurs minimisaient leurs difficultés avant l'entrée dans le groupe afin de ne pas accroître le stress parental et donner l'impression d'être le «bon » enfant dans la famille, tandis que certains parents ont pu surinvestir l'intervention avec une majoration des effets estimés en fin de dispositif. Pour contourner ce biais d'évaluation, le recours à des évaluateurs externes peut être envisagé (enseignants ou tiers) tout comme des stratégies d'observations indirectes avec par exemple l'observation de la relation fraternelle au moyen d'interactions enregistrées sur bandes vidéo (Kryzak et al., 2015).

Au-delà des questionnaires et échelles de mesure utilisés, l'examen en profondeur des profils de réponses qui se dégagent des données qualitatives pourraient permettre d'obtenir des évaluations plus fines de ces programmes. A titre d'exemple, l'analyse du contenu des échanges au fil des séances, au-delà d'une prise en compte des sujets abordés, des émotions et sentiments 
exprimés (Gettings et al., 2015), pourraient inclure la nature des interactions au sein du groupe en fonction des supports utilisés. Inversement, dans les groupes fratries initiés dans une démarche psychodynamique, l'usage d'outils standardisés pour croiser données quantitatives et données qualitatives reste à penser.

\subsubsection{Durée des dispositifs et données de suivi}

La durée des dispositifs, la durée de participation des sujets aux dispositifs (selon qu'ils participent ou non à l'intégralité des interventions), tout comme la difficulté à établir des données de suivi sont des limites majeures.

Comme le soulignent Tudor et Lener (2015), les données les plus solides sur un plan empirique proviennent des études fondées sur des comparaisons entre groupes de traitement et groupes témoins et de celles qui comprennent des données de suivi. Or, très peu d'études proposent ce type de protocole actuellement, la plus récente étant celle de Brouzos et al. (2017). Les modalités de constitution d'un groupe contrôle ne sont d'ailleurs pas toujours précisées - liste d'attente avant intervention (Roberts et al., 2015), affectation aléatoire dans les groupes (Jones et al., 2020) - ce qui limite la possibilité de généraliser les résultats.

\subsubsection{Ancrage théorique}

Au-delà de ces limites méthodologiques, il importe de mentionner le manque d'ancrage théorique des dispositifs mis en place. En effet, peu d'éléments théoriques concernant les modalités d'intervention au sein des groupes sont fournis à travers les différentes études. Exceptés les travaux menés dans une approche psychodynamique qui explicitent le cadre théorique au fondement du mode d'animation adopté pour rendre compte de la dynamique de groupe impulsée (Dayan et al., 2017 ; Despars et al., 2013 ; Milman, 2007 ; Scelles et al., 2012), l'ancrage théorique est le plus souvent déduit à partir de la nature des objectifs mentionnés par les auteurs qui décrivent une approche psychoéducative sans rendre compte spécifiquement des arguments théoriques sous-jacents qui justifient les modalités d'action. Aussi, quelle que soit la démarche entreprise, l'analyse des processus à l'œuvre dans le fonctionnement de ces dispositifs apparaît essentielle pour rendre compte des effets différenciés de la participation à un groupe fratrie selon le cadre mis en place. 


\subsection{Pistes de recherche}

En réponse à ces différentes limites, les auteurs énoncent plusieurs pistes de recherche d'ordre empirique et/ou clinique dans le but d'approfondir la compréhension du lien familial/fraternel en contexte de handicap/maladie et d'améliorer les dispositifs de groupe fratrie. Ces pistes permettent d'entrevoir un enrichissement des modèles d'évaluation des dispositifs et une ouverture du paradigme pour penser la problématique fraternelle.

\subsubsection{Construction de modèles d'évaluation plus robustes}

A partir des différents articles recensés, plusieurs paramètres peuvent être considérés pour améliorer la construction des études.

Tout d'abord, d'autres facteurs d'influence seraient à prendre en compte dans l'étude des effets des dispositifs. Outre l'âge des participants, la nature du handicap/maladie de l'enfant porteur, la sévérité de ses symptômes, certains auteurs insistent sur la nécessité d'évaluer au préalable le niveau d'inadaptation des enfants au développement typique pour ajuster au mieux les interventions (Kryzak et al., 2005) et l'intérêt d'inclure dans l'analyse l'influence de l'écart d'âge dans la fratrie et des rangs de naissance (Brouzos et al., 2017). Les caractéristiques relatives à la famille, telles que le revenu familial, le stress parental, la santé mentale des parents, le parcours de vie de la famille (Jones et al. 2020), sont également des variables non négligeables peu prises en compte. Par ailleurs, même si l'origine ethnique des familles est parfois considérée, aucune étude ne fait état des biais culturels potentiels, alors que l'impact du handicap / de la maladie sur le vécu familial peut varier considérablement selon la culture (Scelles, Ciccone, Korff-Sausse, Missonnier, Salbreux, 2013). Les modèles occidentaux des dispositifs nécessiteraient d'être adaptés pour répondre aux besoins spécifiques de certaines familles et/ou pour être transférables dans d'autres milieux culturels. Aussi, au-delà de la durée des interventions souvent limitées, l'effet de l'espacement des séances sur le processus thérapeutique mériterait également d'être apprécié (Brouzos et al. 2017), tout comme l'effet des lieux d'intervention (Tudor \& Lerner, 2015) pour pouvoir dégager des préconisations plus précises selon les besoins des participants.

Au niveau des protocoles d'évaluation, une meilleure cohérence dans le choix des outils utilisés pourrait favoriser la comparabilité des résultats entre les différentes recherches. $\mathrm{Au}$ delà de l'étude des effets des dispositifs, l'évaluation des processus thérapeutiques en jeu nécessite d'être davantage considérée. Certains travaux non pris en compte dans cette revue de littérature, ne faisant pas état des effets du dispositif observé, peuvent être mentionnés ici au 
titre de leurs perspectives innovantes. C'est le cas par exemple de l'étude de Gabriel (2020), dans une approche psychodynamique, qui porte sur le processus narratif dans les groupes fratries utilisant le dessin comme support d'expression. Ses travaux introduisent une méthodologie originale pour objectiver l'évolution personnelle de chacun des participants à partir de l'analyse des procédés d'élaboration du discours utilisés par les enfants pour « raconter » leurs dessins, sur la base de la grille initialement établie dans le cadre des épreuves projectives (Shentoub, 1996, cité par Gabriel, p. 132). Poursuivi dans une démarche d'évaluation systématique, ce type d'investigation pourrait contribuer non seulement à soutenir les capacités d'élaboration des frères et sœurs mais aussi à mieux appréhender les processus à l'œuvre dans ce type de dispositif.

Par ailleurs, concernant les plans de recherche, il importe de souligner la nécessité d'augmenter la taille des échantillons des groupes d'études et des groupes témoins pour améliorer l'efficacité des études dans une approche empirique. Dans une perspective clinique, la mise en œuvre d'études longitudinales pour déterminer si les groupes fratries favorisent « un développement sain continu et de meilleures relations entre frères et sœurs à long terme » (McCullough \& Simon, 2011, p.323) pourrait permettre notamment de contrer les limites liées à la taille des échantillons et à la nature qualitative des données analysées.

\subsubsection{Paradigme intégrant l'ensemble de la dynamique familiale et perspective life-span}

Plusieurs études mentionnent l'intérêt d'inclure la famille dans le dispositif que ce soit à titre préventif, évaluatif ou informatif (Valls, 2009 ; Roberts et al., 2015 ; Tsao et al., 2012 ; Wallace et al., 2014 ; Wu et al., 2010). Un dispositif à l'attention des parents peut être créé en parallèle à celui de la fratrie voire inclure l'ensemble des membres de la famille dans le but d'agir directement sur l'ensemble de la dynamique familiale. Certains auteurs vont jusqu'à dire que la présence d'un autre enfant sans pathologie au sein de la fratrie a un effet positif sur le bien-être et la gestion du stress parental (Rattaz, Alcaraz \& Baghdadli, 2015). Or peu de travaux prennent en compte les frères et sœurs comme facteur " protecteur » au même titre que le soutien social ou d'autres facteurs environnementaux largement étudiés dans la littérature, ce qui amène à considérer cette dimension dans les études ultérieures.

Enfin, quelles que soient les circonstances, le lien fraternel perdure tout au long de la vie. Aussi, au fil du développement, de nouvelles questions peuvent apparaître sur la place et le rôle à occuper dans la vie du frère/de la sœur porteur de handicap/maladie (Scelles et al. 2012). Des groupes de jeunes adultes ont pu être mis en place mais restent plus rares. Aussi, il 
s'avère opportun d'élargir ces groupes à tous les âges de la vie (Valls, 2019). L'entrée dans 1'âge adulte peut être une période propice pour analyser de manière rétrospective le vécu auprès $\mathrm{du}$ frère / de la sœur en situation de handicap, dans une position davantage extérieure à la famille. Cette étape de la vie se caractérise par la concrétisation de choix professionnel et affectif, et par le projet de donner soi-même naissance à un enfant, différentes tâches développementales qui peuvent être imprégnées du vécu fraternel infantile (Claudel-Valentin, 2018).

\section{Conclusion}

Après ce tour d'horizon des différents travaux scientifiques publiés sur les groupes fratries depuis 2006, il apparaît essentiel de poursuivre la recherche afin de pouvoir mieux asseoir le cadrage théorique et méthodologique de ce type de dispositif. Plusieurs questionnements restent en suspens : comment favoriser la lisibilité des dispositifs et lever les résistances parentales ? quels critères d'inclusion/exclusion poser pour ne pas outrepasser les besoins et capacités de gestion émotionnelle des frères et sœurs ? comment penser la composition des groupes pour s'adapter au mieux aux besoins de l'ensemble des participants? quelle est l'influence du cadre du dispositif sur l'émergence des processus groupaux ? comment comprendre les processus différenciés qui se jouent dans les groupes selon l'âge des participants ? etc.

Ce type de pratique exige prudence et vigilance du côté des praticiens afin de ne pas entrer dans une systématisation qui irait à l'encontre de la prise en compte singulière de chaque situation. Tous les frères et sœurs d'enfants porteurs de handicap/maladie ne développent pas de troubles particuliers en lien avec leur statut spécifique. La cause d'un éventuel mal-être n'est pas forcément directement liée à la situation de handicap. A noter par ailleurs que des effets iatrogènes peuvent parfois s'opérer en pointant trop spécifiquement les difficultés des frères et sœurs avec le risque d'accroître le dysfonctionnement de la fratrie (Tudor et Lener, 2015). La posture de jeunes aidants des frères et sœurs à l'égard de leur pair, généralement non identifiée ou considérée en tant que processus d'apprentissage et d'autonomie par la famille (Jarrige, Dorard \& Untas, 2019) est également à considérer au vu des répercussions que peut engendrer une relation fraternelle asymétrique tant pour les frères et sœurs que pour l'enfant porteur de handicap/maladie (Dayan, 2017). Aussi, les propositions d'accompagnement, si elles s'avèrent 
nécessaires, doivent pouvoir être envisagées au cas par cas. De même le processus d'aide doit pouvoir être suffisamment souple, et les indications de suivi être revues en fonction de l'évolution de l'enfant, ce qui implique une bonne compréhension de la problématique fraternelle dans ces familles.

L'analyse du fonctionnement des groupes fratries en contexte de TSA fait émerger la spécificité des comportements défis et difficultés de communication auxquels sont confrontés les frères et sœurs. L'association de handicap intellectuel avec les TSA amplifie l'impact sur la qualité de vie de la famille / fratrie. Dans ce cadre, une recherche-action ${ }^{1}$ est actuellement en cours, en lien avec plusieurs Centres de Ressources Autisme et structures de soins de la Région Grand-Est, dans le but de prendre en compte des familles confrontées à des troubles autistiques de degrés divers. Associant chercheurs et praticiens, le but est d'établir une évaluation rigoureuse d'un dispositif « groupe fratrie » mis en œuvre dans une approche psychodynamique et systémique auprès d'enfants et d'adolescents sur une durée minimum de deux ans. Par une méthodologie mixte combinant outils cliniques et projectifs et outils standardisés pour rendre compte des effets sur le développement psychoaffectif des participants et la dynamique de leur famille au fil du dispositif, ainsi qu'une analyse des pratiques d'animation permettant une modélisation de l'action, il s'agit de mettre en évidence les besoins développementaux des frères et sœurs selon le degré de sévérité de l'autisme et de créer un guide technique adapté à ce contexte atypique à destination des professionnels engagés dans ce type de dispositif.

Aussi, au vu de l'accroissement des intérêts sociologique et technique pour le domaine numérique (Tichon, 2015), un modèle permettant d'offrir un soutien supplémentaire plus informel après ce type d'intervention, tel qu'un forum internet, pourrait faire l'objet d'une étude complémentaire afin de prendre en compte la pertinence de développer des supports interactifs en ligne, notamment à destination des adolescents et jeunes adultes susceptibles de s'en saisir plus facilement.

Conflit d'intérêt : aucun

\footnotetext{
${ }^{1}$ Recherche intitulée " Groupes fratries de personnes avec TSA : effets sur le vécu psychologique des participants et la qualité des relations intrafamiliales selon les modalités du dispositif », dirigée par S. Claudel-Valentin, soutenue par le Comité national de Coordination de l'Action pour le Handicap (CCAH) et financée par le groupe Humanis Retraite Agirc-Arrco (groupe MMH).
} 


\section{Références}

Brouzos, A., Vassilopoulos, S.P. \& Tassi, C. (2017). A psychoeducational group intervention for siblings of children with autism spectrum disorder. The Journal for Specialists in Group Work, 42(4), 274-298.

Claudel S., Claudon P. \& Coutelle R. (2013). Représentation de soi chez des adolescents ayant un frère ou une sœur autiste. Neuropsychiatrie de l'enfance et de l'adolescence, 61, 39-50.

Claudel-Valentin S. (2018). Construction identitaire des frères et sœurs de personnes avec autisme : genèse du processus d'adultisation dans un contexte atypique. Bulletin de Psychologie, 554, 579-592.

Cooke, J. \& Semmens, C. (2010). The development and evaluation of a support group for siblings of children on the autism spectrum. Good Autism Practice, p.24-29.

Coutelle, R., Pry, R. \& Sibertin-Blanc, D. (2011). Développement et qualité de vie des frères et sœurs adolescents de sujets porteurs d'autismes: une étude pilote. La psychiatrie de l'enfant, 54(1), 201-252.

Dayan, C., Picon, I., Scelles, R., \& Bouteyre, E. (2006). Groupes pour les frères et sœurs d'enfant malade ou handicapé : état de la question. Pratiques psychologiques, 12(2), 221-238.

Dayan, C. \& Scelles, R. (2017). La fratrie face au handicap. Spirale, 1, 70-78.

Dayan, C. (2017). La relation d'aide dans une fratrie avec une personne handicapée. Dialogue, $216,39-51$.

Despars, J., Jaunin, L., Monnier, M., Wannaz, M., Vernez, S., Tolsa, J., Ansermet, F. \& MüllerNix, C. (2013). Un groupe d'accueil pour la fratrie lors d'une naissance à risque dans un service de néonatologie. Devenir, 25(4), 233-243. doi :10.3917/dev.134.0233.

Gabriel, E. (2020). Accompagnement du processus narratif dans un groupe fratrie. Dialogue, 227, 125-141.

Gettings, S., Franco, F., \& Santosh, P. J. (2015). Facilitating support groups for siblings of children with neurodevelopmental disorders using audio-conferencing: a longitudinal feasibility study. Child and adolescent psychiatry and mental health, 9(1), 8. doi: 10.1186/s13034-015-0041-z.

Granat, T., Nordgren, I., Rein, G. \& Sonnander, K. (2012). Group intervention for siblings of children with disabilities: A pilot study in a clinical setting. Disability and Rehabilitation, 34(1), 69-75.

Hartling, L., Milne, A., Tjosvold, L., Wrightson, D., Gallivan, J. et Newton, A. S. (2010). A systematic review of interventions to support siblings of children with chronic illness or disability. Journal of Pediatrics and Child Health, 10, 1-13. 
Hastings, R.P. (2007). Longitudinal relationships between sibling behavioral adjustment and behavior problems of children with developmental disabilities. Journal of Autism and Developmental Disabilities (37), 1485-1492.

Jarrige, E., Dorard, G., Untas, A. (2019). Revue de littérature sur les jeunes aidants : qui sontils et comment les aider? Pratiques psychologiques. doi :10.1016/j.prps.2019.02.003.

Jones, E, Fiani, T., Stewart, J., Neil, N., McHugh, S., Fienyp, D. (2020). Randomized controlled trial of a sibling support group: mental health outcome for siblings of children with autism. Autism, 1-14. doi.org/10.1177/1362361320908979.

Knauss, W. (2009). Le groupe comme thérapeute. Pourquoi faut-il un analyste de groupe ?. Revue de psychothérapie psychanalytique de groupe, 52(1), 31-43. doi:10.3917/rppg.052.0031.

Kryzak, L. A., Cengher, M., Feeley, K. M., Fienup, D. M. \& Jones, E. A. (2015). A community support program for children with autism and their typically developing siblings: Initial investigation. Journal of Intellectual Disabilities, 19(2), 159-177.

Lefebvre des Noëttes, A. \& Sarfaty, J. (2008). La prise en charge de la souffrance psychique de la fratrie dans un groupe d'expression. Dans C. Bert (Ed.) La fratrie à l'épreuve du handicap (pp. 209-218). Toulouse : ERÈS. doi:10.3917/eres.bert.2008.01.0209.

McCullough, K. \& Simon, S. R. (2011). Feeling heard: A support group for siblings of children with developmental disabilities. Social Work with Groups, 34(3-4), 320-329.

Milman, D. (2007). Face au traumatisme de l'enfant sain: des groupes de parole pour frères et sœurs d'enfants atteints de cancer. La psychiatrie de l'enfant, 50 (2), 434-455.

Nillama, V., Derguy, C., Bellalou, L., \& Cappe, É. (2019). Vécu psychologique d'adolescents ayant un frère ou une sœur porteur d'un Trouble du Spectre de l'Autisme et qualité des relations intrafamiliales. Annales Médico-psychologiques, 177(2), 149-156.

Rattaz, C., Alcaraz, C. \& Baghdadli, A. (2016). Evaluation des effets d'un groupe d'accompagnement parental sur le stress et la qualité de vie après l'annonce du diagnostic de trouble du spectre autistique (TSA) chez leur enfant. Annales Médico-Psychologiques, 174, 644-650.

Roberts, R.M., Ejova, A., Giallo, R., Strohm, K., Lillie, M. \& Fuss, B. (2015). A controlled trial of the SibworkS group program for siblings of children with special needs. Research in developmental disabilities, 43-44, 21-31.

Ross, P. \& Cuskelly, M. (2006). Adjustment, sibling problems and coping strategies of brothers and sisters of children with autistic spectrum disorder. Journal of Intellectual and Developmental Disability, 31(2), 77-86.

Scelles, R., Bouteyre, E., Dayan, C. \& Picon, I. (2012). Support groups for sisters and brothers of children with intellectual and developmental disabilities. Early Child Development and Care, 182:2, 249-261, DOI: 10.1080/03004430.2011.553679 
Scelles, R., Ciccone, A., Korff-Sausse, S., Missonnier, S., Salbreux, R. (2013). Famille, culture, handicap. Toulouse : Erès.

Tichon, J. G. (2015). Exploring how children express feelings and emotions in an online support group. Computers in Human Behavior, 53, 469-474.

Tsao, L. L., Davenport, R. \& Schmiege, C. (2012). Supporting siblings of children with autism spectrum disorders. Early Childhood Education Journal, 40(1), 47-54.

Tudor, M. E. \& Lerner, M. D. (2015). Intervention and support for siblings of youth with developmental disabilities: A systematic review. Clinical Child and Family Psychology Review, 18(1), 1-23.

Valls, C. G. (2009). Groups for siblings of children with down syndrome. International medical journal on Down Syndrome, 13(3), 44-47.

Vatne, T.M. \& Zahl, E. (2017). Emotional communication in support groups for siblings of children with disabilities. Patient Education and Counseling, 100, 2106-2108.

Wallace, J., Packman, W., Huffman, L. C., Horn, B., Cowan, M., Amylon, M. D. \& Moses, J. (2014). Psychosocial changes associated with participation in art therapy interventions for siblings of pediatric hematopoietic stem cell transplant patients. Art Therapy, 31(1), 4-11.

Wu., Y, Prout., R, Parikshak. M \& Amylon, S. (2011). Assessing Experiences of Children Who Attended a Camp for Children with Cancer and Their Siblings: A Preliminary Study. Child and Youth Care Forum, 40(2), 121-133. 


\section{Annexe : Analyse des articles retenus}

\begin{tabular}{|c|c|c|c|c|c|c|c|}
\hline $\mathbf{N}^{\circ}$ & $\begin{array}{l}1^{\text {er }} \text { Auteur } \\
\text { (année) }\end{array}$ & Pays & $\begin{array}{c}\text { Type d'intervention } \\
\text { (modèle associé, objectifs, } \\
\text { durée, lieu) }\end{array}$ & $\begin{array}{l}\text { Handicaps/ } \\
\text { Maladies } \\
\text { considérés }\end{array}$ & $\begin{array}{c}\text { N/Age } \\
\text { des frères et } \\
\text { sœurs }\end{array}$ & $\begin{array}{c}\text { Évaluation } \\
\text { (outils utilisés, temporalité) }\end{array}$ & Effets observés \\
\hline 1 & Brouzos (2017) & GRC & $\begin{array}{l}\text { Groupe psychoéducatif } \\
\text { - Approche cognitivo- } \\
\text { comportementale centrée sur les } \\
\text { troubles de l'enfant TSA, } \\
\text { l'adaptation émotionnelle et } \\
\text { sociale, la restructuration } \\
\text { cognitive, la résolution de } \\
\text { problème } \\
\text { - } 8 \text { séances hebdomadaires d' } 1 \mathrm{~h} 30 \\
\text { - Centre d'activités pour enfants } \\
\text { handicapés }\end{array}$ & TSA & $\begin{array}{l}38 / 6-15 \text { ans } \\
\text { - Gr. expé }: 22 \\
* 6 \text { ans }: 5 \\
* 8-10 \text { ans }: 5 \\
* 11-12 \text { ans }: 7 \\
* 14-15 \text { ans }: 5 \\
\text { - Gr. témoin :16 }\end{array}$ & $\begin{array}{l}\text { - Questionnaires auto-rapportés : } \\
\text { * Knowledge of Autism Syndrome } \\
\text { (Ross \& Cuskelly, 2006); } \\
\text { * Echelle d'adaptation et de } \\
\text { rajustement (Perry, 1989); } \\
\text { * Strengths and Difficulties } \\
\text { Questionnaire (Goodman, Meltzer, \& } \\
\text { Bailey, 1998) } \\
\text { A T1 et T2 = } 8 \text { semaines }\end{array}$ & $\begin{array}{l}\text { - Augmentation significative } \\
\text { des connaissances sur les TSA } \\
\text { dans le GE (davantage marqué } \\
\text { chez les enfants) } \\
\text { - Réduction significative des } \\
\text { difficultés d'adaptation et des } \\
\text { problèmes émotionnels et } \\
\text { comportementaux dans le GE } \\
\text { (davantage marqué chez les } \\
\text { adolescents) }\end{array}$ \\
\hline 2 & Cooke (2010) & GBR & $\begin{array}{l}\text { Groupe de soutien } \\
\text { - Approche psycho-éducative } \\
\text { visant à explorer les conditions de } \\
\text { vie des frères et sœurs et leurs } \\
\text { possibilités de composer avec } \\
\text { l'autisme } \\
\text { - } 8 \text { séances hebdomadaires d'1h30 } \\
\text { - Association médico-sociale } \\
\text { (Ecole Norsaca) }\end{array}$ & TSA & $12 / 8-12$ ans & $\begin{array}{l}\text { - Questionnaire hétéro-rapporté } \\
\text { (parents) : Info démographiques, } \\
\text { compréhension du frère/de la sœur } \\
\text { sur les TSA, préoccupations } \\
\text { familiales, attentes par rapport au } \\
\text { dispositif } \\
\text { A T1 et T2 = } 1 \text { semaine après la fin } \\
\text { du dispositif } \\
\text { - Réalisation d'une affiche par les } \\
\text { frères et sœurs sur leurs } \\
\text { connaissances concernant les TSA } \\
\left(1^{\text {ere }} \text { et } 7^{\text {ème }} \text { séance) }\right. \\
\end{array}$ & $\begin{array}{l}\text { - Satisfaction parentale : } \\
\text { meilleure compréhension et } \\
\text { adaptation de la fratrie }\end{array}$ \\
\hline 3 & Dayan (2017) & FRA & $\begin{array}{l}\text { Groupe de parole } \\
\text { - Approche psychodynamique } \\
\text { (libre association autour du } \\
\text { handicap) } \\
\text { - Séances mensuelles d'une heure } \\
\text { - Lieu de soin (CAMSP) }\end{array}$ & $\begin{array}{c}\text { Handicaps } \\
\text { (troubles neuro- } \\
\text { développementaux) }\end{array}$ & $6 / 5-8$ ans & $\begin{array}{l}\text { - Entretien préalable avec les } \\
\text { parents et l'enfant concerné } \\
\text { - Observation directe au fil des } \\
\text { séances } \\
\text { - Entretien avec les parents après } 10 \\
\text { séances }\end{array}$ & $\begin{array}{l}\text { - Conscientisation du handicap } \\
\text { - Positionnement et affirmation } \\
\text { au sein de la famille }\end{array}$ \\
\hline
\end{tabular}




\begin{tabular}{|c|c|c|c|c|c|c|c|}
\hline 4 & Despars (2013) & SUI & $\begin{array}{l}\text { Groupe de parole } \\
\text { - Approche } \\
\text { psychodynamique (libre- } \\
\text { association autour de la naissance } \\
\text { et de l'hospitalisation) } \\
\text { - Séances hebdomadaires d'1h30 } \\
\text { - Espace spécifique dans l'hôpital } \\
\text { en dehors de l'unité } \\
\text { d'hospitalisation }\end{array}$ & $\begin{array}{c}\text { Enfants hospitalisés } \\
\text { en néonatalogie }\end{array}$ & $\begin{array}{l}\text { A partir de } 24 \\
\text { mois }\end{array}$ & $\begin{array}{l}\text {-Questionnaire hétéro-rapporté } \\
\text { (parents) : manifestations } \\
\text { comportementales et } \\
\text { psychosomatiques observées chez } \\
\text { les frères et sœurs (avant l'entrée } \\
\text { dans le dispositif) } \\
\text { - Observation directe au fil des } \\
\text { séances }\end{array}$ & $\begin{array}{l}\text { - Représentations plus précises } \\
\text { de la situation de l'enfant } \\
\text { hospitalisé } \\
\text { - Élaboration des angoisses, } \\
\text { sentiment de jalousie, } \\
\text { culpabilité } \\
\text { - Repérage des situations à } \\
\text { risque }\end{array}$ \\
\hline 5 & Gettings (2015) & GBR & $\begin{array}{l}\text { Groupe de soutien } \\
\text { - Intervention centrée sur la } \\
\text { compréhension des troubles, les } \\
\text { conditions de vie familiales et } \\
\text { extra-familiales, la projection } \\
\text { dans l'avenir } \\
\text { - } 8 \text { séances hebdomadaires d'1h : } \\
4 \text { séances en présentiel, } 4 \text { par } \\
\text { audioconférence } \\
\text { - Lieu de soin (Centre for } \\
\text { Interventional } \\
\text { Paediatric Psychopharmacology) }\end{array}$ & $\begin{array}{c}\text { Troubles neuro- } \\
\text { Développementaux } \\
\text { complexes } \\
\text { (comorbidité) }\end{array}$ & $6 / 8-13$ ans & $\begin{array}{l}\text { - A T1, questionnaire hétéro- } \\
\text { rapporté (parents) : Profil des } \\
\text { symptômes neuropsychiatriques } \\
\text { - A T1 et T2 (juste après } \\
\text { l'intervention), questionnaire auto- } \\
\text { rapporté sur les forces et } \\
\text { difficultés (fonctionnement affectif } \\
\text { et comportemental) et entrevue } \\
\text { semi-structurée avec les frères et } \\
\text { sœurs (comportements de l'enfant } \\
\text { porteur de troubles, impact du } \\
\text { comportement sur la famille, besoin } \\
\text { d'en parler) } \\
\text { - Entrevue non structurée avec la } \\
\text { fratrie et/ou les parents à T3 (3 à } 6 \\
\text { mois après l'intervention) } \\
\text { - Séances enregistrées et } \\
\text { retranscrites (analyse thématique) }\end{array}$ & $\begin{array}{l}\text { - Acceptabilité de } \\
\text { l'audioconférence } \\
\text { - Diminution de la gravité des } \\
\text { préoccupations } \\
\text { - Communication accrue } \\
\text { - Réseau social élargi } \\
\text { - Augmentation des } \\
\text { compétences en résolution de } \\
\text { problème } \\
\text { - Renforcement de la résilience }\end{array}$ \\
\hline 6 & Granat (2012) & SWE & $\begin{array}{l}\text { Groupe d'intervention } \\
\text { - Approche psycho-éducative } \\
\text { visant à accroître les } \\
\text { connaissances sur le handicap, } \\
\text { fournir des outils de résolution de } \\
\text { problème pour gérer plus } \\
\text { efficacement la relation fraternelle }\end{array}$ & $\begin{array}{c}\text { TSA, TDAH, } \\
\text { déficience } \\
\text { intellectuelle } \\
\text { déficience physique }\end{array}$ & $54 / 8-12$ & $\begin{array}{l}\text {-Questionnaires auto-rapportés } \\
\text { avant et après l'intervention : } \\
\text { *Sibling Knowledge Interview (SKI, } \\
\text { Lobato \& Kao, 2002) } \\
\text { *Sibling Relationship } \\
\text { Questionnaires (SRQ, Buhrmester \& } \\
\text { Furman, 1990) }\end{array}$ & $\begin{array}{l}\text { - Meilleures connaissances des } \\
\text { troubles pour l'ensemble des } \\
\text { sujets } \\
\text { - Résultats contrastés sur le } \\
\text { plan adaptatif selon la nature } \\
\text { des troubles, aucun } \\
\text { changement significatif }\end{array}$ \\
\hline
\end{tabular}




\begin{tabular}{|c|c|c|c|c|c|c|c|}
\hline & & & $\begin{array}{l}\text { - } 6 \text { séances hebdomadaires de } 2 \\
\text { heures sur } 18 \text { mois } \\
\text { - Centre d'habilitation } \\
\text { ambulatoire }\end{array}$ & & & & $\begin{array}{l}\text { concernant les stratégies de } \\
\text { résolution de problème pour } \\
\text { les frères et sœurs d'enfants } \\
\text { TSA }\end{array}$ \\
\hline 7 & Jones (2020) & USA & $\begin{array}{l}\text { Groupe de soutien } \\
\text { - Triple dispositif : groupe de } \\
\text { soutien pour les frères et sœurs } \\
\text { (versus groupe de contrôle social), } \\
\text { interventions sur la } \\
\text { communication et les } \\
\text { compétences sociales des enfants } \\
\text { TSA, espace récréatif pour } \\
\text { l'ensemble de la fratrie } \\
\text { - Approche psychoéducative } \\
\text { visant l'expression de sentiments } \\
\text { liés au frère ou à la sœur atteint(e) } \\
\text { de TSA et à sa famille, la } \\
\text { résolution de problèmes, les } \\
\text { capacités d'adaptation, } \\
\text { l'élargissement d'un réseau de } \\
\text { pairs, l'information concernant les } \\
\text { TSA } \\
\text { - 2 heures par semaine pendant } 10 \\
\text { semaines (1h groupes } \\
\text { différenciés, lh fratrie réunie) } \\
\text { - Milieu universitaire }\end{array}$ & TSA & $\begin{array}{l}54 \\
\text { - Gr. de soutien : } \\
30 \text { (4-15 ans) } \\
\text { - Gr. de contrôle } \\
\text { social (centré } \\
\text { uniquement sur } \\
\text { l'attention) : } 24 \text { (3- } \\
18 \text { ans) }\end{array}$ & $\begin{array}{l}\text { Mesures pré (envoi 3-4 semaines } \\
\text { avant le programme, retour 1ère } \\
\text { séance) post-intervention (envoi } \\
9^{\text {ème }} \text { semaine du programme, retour } \\
\text { à la dernière séance) } \\
\text { - Mesures auto-rapportées (frères et } \\
\text { sœurs) : } \\
\text { * Children's Depression Inventory- } \\
\text { 2nd Edition (CDI-2; Kovacs, 2011); } \\
\text { * Revised Children Manifest } \\
\text { Anxiety Scale-Second Edition } \\
\text { (RCMAS-2 ; Reynolds \& Richmond, } \\
\text { 2008); } \\
\text { * questionnaire sur le réseau de } \\
\text { soutien; } \\
\text { * liste de contrôle des stratégies } \\
\text { d'adaptation des enfants (Ayers et } \\
\text { al., 1996) } \\
\text { - Mesures hétéro-rapportées } \\
\text { (parents) : } \\
\text { * Child Behavior Checklist (Achen- } \\
\text { bach et Rescorla, 2000, 2001) pour } \\
\text { chaque membre de la fratrie } \\
\text { + Evaluation du degré de TSA (3 } 3^{\text {ème }} \\
\text { séance) avec échelle d'évaluation de } \\
\text { l'autisme infantile (CARS-II, } \\
\text { Schopler, 2010) }\end{array}$ & $\begin{array}{l}\text { - Amélioration globale dans } \\
\text { les deux groupes } \\
\text { - Amélioration du } \\
\text { comportement } \\
\text { d'externalisation et des } \\
\text { capacités d'adaptation des } \\
\text { frères et sœur } \\
\text { significativement plus } \\
\text { importante dans le groupe de } \\
\text { soutien } \\
\text { - Dans le cas de TSA avec } \\
\text { symptômes sévères, le groupe } \\
\text { de soutien peut servir de } \\
\text { tampon contre les symptômes } \\
\text { d'anxiété et de dépression des } \\
\text { frères et sœurs } \\
\text { - Pas de changement } \\
\text { concernant le réseau de soutien }\end{array}$ \\
\hline 8 & Kryzak (2015) & USA & $\begin{array}{l}\text { Programme d'intervention } \\
\text { - Triple dispositif : groupe de } \\
\text { soutien pour les frères et sœurs, } \\
\text { intervention sur les compétences }\end{array}$ & $\begin{array}{c}\text { TSA } \\
\text { (avec ou sans } \\
\text { comorbidités) }\end{array}$ & $15 / 4-14$ ans & $\begin{array}{l}\text {-Questionnaires auto-rapportés } \\
\text { (avant /après l'intervention) } \\
\text { * Child Depression Inventory (IDC, } \\
\text { Kovacs, 1985) }\end{array}$ & $\begin{array}{l}\text { - Diminution de la dépression } \\
\text { et de l'anxiété }\end{array}$ \\
\hline
\end{tabular}




\begin{tabular}{|c|c|c|c|c|c|c|c|}
\hline & & & $\begin{array}{l}\text { des enfants TSA, espace de loisir } \\
\text { pour l'ensemble de la fratrie } \\
\text { - Approche psychoéducative } \\
\text { visant les connaissances au sujet } \\
\text { des TSA, le développement d'un } \\
\text { réseau de pairs, l'adaptation et les } \\
\text { interactions dans la fratrie } \\
\text { - } 8 \text { séances hebdomadaires de } 2 \mathrm{~h} \\
\text { - Établissements universitaires }\end{array}$ & & & $\begin{array}{l}\text { *Revised Children Manifest Anxiety } \\
\text { Scale-Second Edition (RCMAS-2, } \\
\text { Reynolds et Richmond, 2008) } \\
\text { * Autism Sibling Knowledge (ASK) } \\
\text { - Observations comportementales } \\
\text { des interactions entre frères et } \\
\text { sœurs (enregistrements vidéos } \\
\text { codés) } \\
\text { - Questionnaire de validité sociale } \\
\text { (parents, frères et sœurs, animateur) } \\
\text { à la fin du programme }\end{array}$ & $\begin{array}{l}\text { - Diminution de l'estime de soi } \\
\text { négative et des problèmes } \\
\text { interpersonnels } \\
\text { - Diminution de l'anxiété } \\
\text { physiologique } \\
\text { - Défenses toujours présentes } \\
\text { - Connaissances sur les TSA } \\
\text { augmentées } \\
\text { - Evolution positive des } \\
\text { interactions fraternelles - } \\
\text { Elargissement du réseau de } \\
\text { pairs }\end{array}$ \\
\hline 9 & $\begin{array}{l}\text { McCullough } \\
(2011)\end{array}$ & USA & $\begin{array}{l}\text { Groupe de soutien (programme } \\
\text { pilote) } \\
\text { - Approche psycho-éducative } \\
\text { (activités récréatives, de partage } \\
\text { d'information et de soutien par } \\
\text { les pairs centrée sur l'entraide) } \\
\text { - Objectifs : développement d'un } \\
\text { réseau de pairs, de } \\
\text { connaissances sur les } \\
\text { déficiences, de stratégies } \\
\text { d'adaptation, de l'estime de soi } \\
\text { - Prestataire privé de services } \\
\text { cliniques aux enfants et adultes } \\
\text { présentant un retard de } \\
\text { développement } \\
\text { - Séance mensuelle de janvier à } \\
\text { mai }\end{array}$ & TSA avec DI & $\begin{array}{l}3 \text { présents } \\
\text { régulièrement /7- } \\
12 \text { ans }\end{array}$ & $\begin{array}{l}\text { - Observation directe au fil des } \\
\text { séances }\end{array}$ & $\begin{array}{l}\text { - Meilleure compréhension de } \\
\text { l'expérience de vie de leur } \\
\text { pair } \\
\text { - Développement des } \\
\text { capacités d'empathie et de } \\
\text { patience } \\
\text { - Communication } \\
\text { intrafamiliale accrue sur } \\
\text { leurs sentiments, rôles } \\
\text { familiaux et comportements }\end{array}$ \\
\hline 10 & Milman (2007) & FRA & $\begin{array}{l}\text { Groupe de parole } \\
\text { - Approche psychodynamique } \\
\text { (Association libre) } \\
\text { - Service d'oncologie pédiatrique }\end{array}$ & Cancer & $\begin{array}{l}74 / 5-11 \text { ans et } \\
11-17 \text { ans }\end{array}$ & $\begin{array}{l}\text { - Entretien individuel préalable } \\
\text { avec l'enfant et les parents centré } \\
\text { sur les difficultés et souffrances } \\
\text { face à la maladie }\end{array}$ & $\begin{array}{l}\text { - Désenclavement de la } \\
\text { solitude (groupe 5-11 ans) } \\
\text { - Levée partielle de la } \\
\text { culpabilité (groupe 5-11 ans) }\end{array}$ \\
\hline
\end{tabular}




\begin{tabular}{|c|c|c|c|c|c|c|c|}
\hline & & & - Durée : 3 ans & & & $\begin{array}{l}\text { - Observation directe au fil des } \\
\text { séances }\end{array}$ & \\
\hline 11 & $\begin{array}{l}\text { Roberts \& al. } \\
(2015)\end{array}$ & AUS & $\begin{array}{l}\text { Programme de groupe de soutien } \\
\text { (SibWorks) } \\
\text { - Intervention cognitivo- } \\
\text { comportementale visant à } \\
\text { promouvoir le fonctionnement } \\
\text { émotionnel et comportemental des } \\
\text { frères et sœurs } \\
\text { - Approche psycho-éducative } \\
\text { axée sur le renforcement du } \\
\text { soutien social perçu, l'estime de } \\
\text { soi, les aptitudes à résoudre les } \\
\text { problèmes, les comportements } \\
\text { d'adaptation et les relations } \\
\text { positives entre frères et sœurs } \\
\text { - } 6 \text { séances de groupe } \\
\text { hebdomadaires de } 2 \text { heures } \\
\text { - Organismes pour personnes } \\
\text { handicapées }\end{array}$ & $\begin{array}{c}\text { Handicaps et } \\
\text { maladies } \\
\text { chroniques (TSA, } \\
\text { Syndrome } \\
\text { d'Angelman, } \\
\text { Syndrome de Down, } \\
\text { Syndrome de Phelan- } \\
\text { McDermid, retard de } \\
\text { développement } \\
\text { global, déficience } \\
\text { intellectuelle et } \\
\text { hypoplasie du nerf } \\
\text { optique) }\end{array}$ & $\begin{array}{l}56 / 7-12 \text { ans } \\
\text { - Groupe expé }= \\
30 \\
\text { (4 groupes de 6- } \\
8 \text { enfants) } \\
\text { - Groupe témoin } \\
=26\end{array}$ & $\begin{array}{l}\text { - Évaluation avant la première } \\
\text { séance du groupe expérimental } \\
\text { (T1), immédiatement après la } \\
\text { dernière séance du groupe } \\
\text { expérimental (T2) et } 3 \text { mois après } \\
\text { la dernière séance du groupe } \\
\text { expérimental (T3) } \\
\text { - Questionnaires hétéro-rapportés } \\
\text { (parents) à T1, T2 et T3 : } \\
\text { *Strenght and Difficulties } \\
\text { Questionnaire - parental version } \\
\text { (SDQ; Goodman, Meltzer, \& Bailey, } \\
\text { 1998) } \\
\text { *Echelle de soutien social pour } \\
\text { enfants : sous-échelles parents, } \\
\text { enseignants, camarades, amis } \\
\text { (SSSC; Harter, 1985) } \\
\text { *Questionnaire sur les relations } \\
\text { entre frères et sæurs (SRQ; } \\
\text { Buhrmester \& Furman, 1990) : } 2 \text { sous- } \\
\text { échelles utilisées : « chaleur et } \\
\text { proximité », « conflit ». } \\
\text { *Echelle d'auto-évaluation de } \\
\text { 1'adaptation (SRCS, Causey \& } \\
\text { Dubow, 1992) } \\
\text { *Rosenberg Self-Esteem Scale } \\
\text { (RSES ; Rosenberg, 1989) } \\
\text { - Questionnaire hétéro-rapporté } \\
\text { (T2) : enquête de satisfaction en } 6 \\
\text { points adaptée (2013) } \\
\text { - Mesure qualitative auto-rapportée } \\
\text { (T2) : énumérer } 3 \text { choses apprises }\end{array}$ & $\begin{array}{l}\text { - Diminution des difficultés } \\
\text { émotionnelles et } \\
\text { comportementales dans le } \\
\text { groupe d'intervention à T2 et } \\
\text { T3. } \\
\text { - Effet sur l'estime de soi et } \\
\text { l'externalisation non } \\
\text { maintenu à T3 } \\
\text { - Surestimation des parents / } \\
\text { frères et sœurs à T1 } \\
\text { - Validation sociale du } \\
\text { programme }\end{array}$ \\
\hline
\end{tabular}




\begin{tabular}{|c|c|c|c|c|c|c|c|}
\hline & & & & & & $\begin{array}{l}\text { pendant l'intervention durant la } \\
\text { dernière séance (réponses } \\
\text { enregistrées pour analyse } \\
\text { thématique) } \\
\text { - Mesures complémentaires (T1) : } \\
\text { * Mémoire Developmental } \\
\text { Behaviour Checklist - P24 (DBC- } \\
\text { P24; Taffe et al., 2007) (hétéro- } \\
\text { rapportée) } \\
\text { *Données démographiques }\end{array}$ & \\
\hline 12 & Scelles (2012) & $\begin{array}{l}\text { FRA } \\
\text { BEL }\end{array}$ & $\begin{array}{l}\text { Groupes de parole } \\
\text { - Mis en œuvre dans } 10 \\
\text { établissements (lieux de soins de } \\
\text { la personne avec handicap : } \\
\text { CAMSP, Sessad) } \\
\text { - En place depuis } 2 \text { ans min. } \\
\text { - Approche psychodynamique }\end{array}$ & Handicaps & $\begin{array}{l}\text { - Non précisé / 4- } \\
15 \text { ans }\end{array}$ & $\begin{array}{l}\text { - Entretien semi-structuré avec } \\
\text { frères et sœurs (6), parents (6), } \\
\text { professionnels (9) } \\
\text { - Groupe de discussion avec les } \\
\text { équipes (6) comprenant } 8 \text { à } 10 \\
\text { professionnels } \\
\text { - Exploration des effets du } \\
\text { dispositif sur les liens fraternels } \\
\text { et les liens entre enfants-parents } \\
\text { - Exploration des indications, } \\
\text { contre-indications et des limites } \\
\text { de ces groupes } \\
\text { - Analyse de contenu thématique } \\
\text { longitudinale et transversale intra } \\
\text { et inter-populations des entretiens } \\
\text { semi-directifs enregistrés. }\end{array}$ & $\begin{array}{l}\text { - Rupture de la solitude } \\
\text { - Favorisation de la parole en } \\
\text { famille (estimation plus forte } \\
\text { des professionnels/familles) } \\
\text { - Apaisement des enfants } \\
\text { grâce aux échanges sur le } \\
\text { handicap et partage } \\
\text { d'expériences } \\
\text { - Meilleure compréhension du } \\
\text { handicap et tolérance } \\
\text { envers l'enfant porteur de } \\
\text { handicap } \\
\text { - Diminution de la culpabilité } \\
\text { des parents } \\
\text { - Meilleure compréhension de } \\
\text { la dynamique famille par les } \\
\text { professionnels }\end{array}$ \\
\hline 13 & Tichon (2015) & AUS & $\begin{array}{l}\text { Groupe de soutien en ligne } \\
\text { "Sibkids » } \\
\text { - Approche psychoéducative } \\
\text { - Durée : } 3 \text { mois }\end{array}$ & $\begin{array}{c}\text { « Besoins } \\
\text { spéciaux » } \\
\text { (handicaps et/ou } \\
\text { maladies) }\end{array}$ & $58 / 7-17$ ans & $\begin{array}{l}\text { - Analyse du contenu des échanges } \\
\text { au sein du groupe à partir de } \\
\text { données quantitatives et } \\
\text { qualitatives (schéma de codage : } \\
\text { sujets abordés, type de soutien } \\
\text { social échangé, nature de l'auto- } \\
\text { divulgation partagée) }\end{array}$ & $\begin{array}{l}\text { - Émotions négatives } \\
\text { discutées plus souvent que } \\
\text { les émotions positives } \\
\text { - Sujets les plus abordés : } \\
\text { problèmes survenant au sein } \\
\text { de la famille }\end{array}$ \\
\hline
\end{tabular}




\begin{tabular}{|c|c|c|c|c|c|c|c|}
\hline & & & & & & & $\begin{array}{l}\text { - Émotions et sentiments les } \\
\text { plus souvent évoqués en lien } \\
\text { avec les interactions entre les } \\
\text { enfants et leur pair porteur, } \\
\text { leurs parents ou en réaction } \\
\text { aux interactions parents-pair } \\
\text { ayant des besoins } \\
\text { spécifiques. } \\
\text { - La technologie n'entrave pas } \\
\text { leur capacité à partager leurs } \\
\text { préoccupations intimes }\end{array}$ \\
\hline 14 & Valls (2009) & ESP & $\begin{array}{l}\text { Groupe de parole } \\
\text { - Objectifs : connaître d'autres } \\
\text { enfants ayant un frère ou une } \\
\text { sœur porteur du SD, mieux } \\
\text { comprendre le SD, être capable } \\
\text { d'exprimer et d'identifier ses } \\
\text { sentiments, partager des } \\
\text { expériences et s'entraider } \\
\text { - Fondation Catalane Syndrome } \\
\text { de Down (FCSD) } \\
\text { - } 3 \text { séances de } 90 \text { minutes }\end{array}$ & Syndrome de Down & $\begin{array}{l}\text { Groupes de } 4 \text { à } 10 \\
\text { enfants/ } \\
\text { Deux tranches } \\
\text { d'âge : } 5-10,11- \\
16 \text { ans }\end{array}$ & $\begin{array}{l}\text { - En fin de séance : réunion entre } \\
\text { enfants/thérapeutes pour discuter } \\
\text { de leurs opinions et de leur } \\
\text { niveau de satisfaction du groupe } \\
\text { - Après chaque séance : réunion } \\
\text { entre parents/thérapeutes pour } \\
\text { discuter des activités et des } \\
\text { questions importantes posées } \\
\text { dans le groupe } \\
\text { - Possibilité d'entretiens } \\
\text { individuels avec le thérapeute } \\
\text { pour les parents qui le souhaitent }\end{array}$ & $\begin{array}{l}\text { - Expérience positive pour la } \\
\text { majorité des enfants } \\
\text { - Effets positifs sur la famille : } \\
\text { changement comportemental } \\
\text { des enfants du point de vue } \\
\text { des parents (plus proche et } \\
\text { plus affectueux du } \\
\text { frère/sœur, plus ouverts, plus } \\
\text { de communication) }\end{array}$ \\
\hline 15 & Vatne (2017) & NOR & $\begin{array}{l}\text { Groupe de soutien } \\
\text { - } 3 \text { séances de discussion ouverte } \\
\text { (troubles fratrie, perception de soi } \\
\text { et relations familiales, émotions). } \\
\text { - Centre de Ressource Norvégien }\end{array}$ & $\begin{array}{c}\text { Handicaps : } \\
\text { (cognitif, physique) }\end{array}$ & $\begin{array}{l}30 / 11-16 \text { ans } \\
6 \text { groupes } \\
\text { appariés selon } \\
\text { 1'âge }\end{array}$ & $\begin{array}{l}\text { - Questionnaire auto-rapporté } \\
\text { (parents) : données } \\
\text { démographiques } \\
\text { - Enregistrement sur bande vidéo } \\
\text { de toutes les séances } \\
\text { - Analyse de contenu des } \\
\text { conversations : fréquence des } \\
\text { expressions et réponses } \\
\text { émotionnelles }\end{array}$ & $\begin{array}{l}\text { - Expression d'émotions } \\
\text { négatives et de } \\
\text { préoccupations ( } 59 \% \\
\text { implicites, } 41 \% \text { explicites) } \\
\text { - Réponses immédiates } \\
\text { fournies par le chef de } \\
\text { groupe dans } 98 \% \text { des cas } \\
\text { (peu de réactions directes des } \\
\text { autres enfants) }\end{array}$ \\
\hline
\end{tabular}




\begin{tabular}{|c|c|c|c|c|c|c|c|}
\hline & & & & & & & $\begin{array}{l}\text { - } 38 \% \text { des réponses portaient } \\
\text { sur l'émotion, la cognition } \\
\text { ou le comportement } \\
\text { - L'âge et le sexe n'expliquent } \\
\text { pas la variation individuelle } \\
\text { de la quantité d'émotions } \\
\text { exprimée }\end{array}$ \\
\hline 16 & Wallace (2014) & USA & $\begin{array}{l}\text { Interventions art-thérapeutiques } \\
\text { visant le bien-être psycho-social } \\
\text { - } 3 \text { séances d'art-thérapie : } 1 \\
\text { semaine, } 1 \text { mois et } 3 \text { mois après } \\
\text { l'intervention HSCT de leur pair } \\
\text { - Durée de l'étude }: 3 \text { ans } \\
\text { - Salle privée de l'hôpital ou tout } \\
\text { autre endroit choisi par la } \\
\text { famille } \\
\text { - Durée variable : } 90 \text { minutes- } \\
\text { 2h00 }\end{array}$ & $\begin{array}{l}\text { Cancers infantiles } \\
\text { ou maladies non } \\
\text { malignes de la } \\
\text { moelle (contexte de } \\
\text { transplantation de } \\
\text { la moelle avec } \\
\text { frères et sœurs) }\end{array}$ & $\begin{array}{l}30 / 6-16 \text { ans } \\
\text { Groupe témoin } \mathrm{N} \\
=10 \\
\text { Groupe } \\
\text { d'intervention } \mathrm{N} \\
=20\end{array}$ & $\begin{array}{l}\text { Mesures à T1 (séance 1), T2 } \\
\text { (séance 2) et T3 (séance 3) } \\
\text { - Questionnaires hétéro-rapportés } \\
\text { (parents) : } \\
\text { * Données démographiques } \\
\text { * Caractéristiques médicales de } \\
\text { l'enfant malade } \\
\text { * McMaster Family Assessment } \\
\text { Device (Epstein, Baldwin \& Bishop, } \\
\text { 1983) } \\
\text { - Questionnaires auto- } \\
\text { rapportés (enfant) : } \\
\text { * Revised Children's Manifest } \\
\text { Anxiety Scale- Second Edition } \\
\text { (RCMAS-2, Reynolds \& Richmond, } \\
\text { 2008) } \\
\text { * Piers-Harris Children's Self- } \\
\text { Concept Scale- Second Edition } \\
\text { (Piers-Harris 2, Piers, Harris, \& } \\
\text { Herzberg, 2002) } \\
\text { * Indice UCLA PTSD for DSM-IV } \\
\text { (UPID ; Pynoos, Rodriguez, } \\
\text { Steinberg, Stuber, \& Frederick, 1998) } \\
\text { (auto- et hétéro-rapportés : parents } \\
\text { et enfant) }\end{array}$ & $\begin{array}{l}\text { - Amélioration significative } \\
\text { des symptômes de stress post- } \\
\text { traumatique auto-rapportés } \\
\text { lors de la séance finale dans le } \\
\text { groupe d'intervention } \\
\text { - Pas de différence } \\
\text { significative entre le groupe } \\
\text { d'intervention et le groupe } \\
\text { témoin concernant le concept } \\
\text { de soi, l'anxiété, le } \\
\text { fonctionnement familial ou } \\
\text { les symptômes de stress } \\
\text { post-traumatique déclarés } \\
\text { par les parents } \\
\text { - Appréciation de } \\
\text { l'intervention par les } \\
\text { participants qui estiment } \\
\text { avoir pu jouer un rôle } \\
\text { important dans le traitement } \\
\text { de leur pair et ressentent } \\
\text { moins de culpabilité et } \\
\text { d'impuissance }\end{array}$ \\
\hline 17 & $\mathrm{Wu}(2011)$ & USA & $\begin{array}{l}\text { Camps d'été (Okizu) pour enfants } \\
\text { ayant un cancer et leurs frères et } \\
\text { sœurs }\end{array}$ & $\begin{array}{l}\text { Maladies } \\
\text { chroniques } \\
\text { (cancers) }\end{array}$ & $\begin{array}{l}73 / 5-18 \text { ans } \\
+ \text { Enfants atteints } \\
\text { de cancer }=56\end{array}$ & $\begin{array}{l}\text { - A T1 (avant le camp) : } \\
\text { *formulaire démographique et } \\
\text { questionnaire portant sur les }\end{array}$ & $\begin{array}{l}\text { - Satisfaction des parents et } \\
\text { des enfants de l'expérience } \\
\text { du camp }\end{array}$ \\
\hline
\end{tabular}




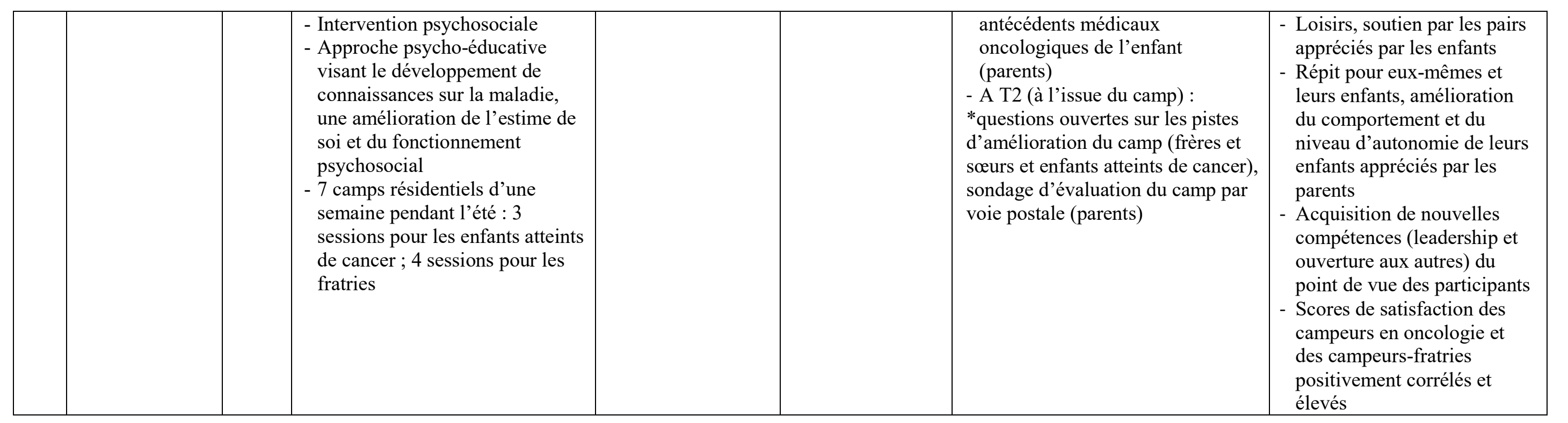

